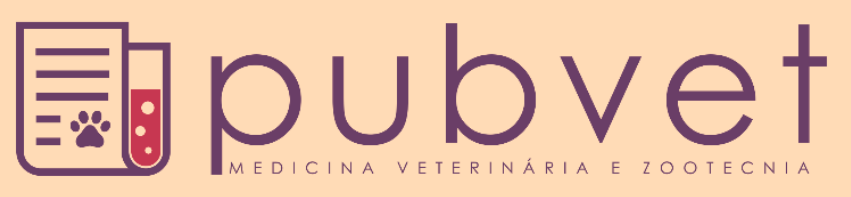

https://doi.org/10.31533/pubvet.v14n10a679.1-4

\title{
Efeito do tempo e da temperatura de armazenamento nos parâmetros hematológicos de cães hígidos
}

\author{
Kamila Líbano de Souza ${ }^{1 *} \bullet$, Margarete Kimie Falbo ${ }^{2} \bullet$ \\ ${ }^{I}$ Residente em Patologia Clínica Veterinária, Universidade Estadual do Centro-Oeste, Departamento de Medicina Veterinária. Guarapuava - PR, Brasil. \\ ${ }^{2}$ Professora da Universidade Estadual do Centro-Oeste, Departamento de Medicina Veterinária. Guarapuava -PR Brasil. \\ *Autor para correspondência, E-mail: kmyla_libano@hotmail.com
}

Resumo. O objetivo deste trabalho foi avaliar a estabilidade dos parâmetros hematológicos em diferentes tempos de armazenamento e temperatura. Utilizou-se sangue total de 7 cães (Canis lupus familiaris), hígidos. As amostras foram divididas em 2 alíquotas de $2 \mathrm{~mL}$ acondicionadas em tubos de EDTA-K3, conservadas em temperatura ambiente a $22^{\circ} \mathrm{C}$ e sob refrigeração $4^{\circ} \mathrm{C}$, nos seguintes intervalos de tempo T0h, T6h, T12h, T24h, T48h. Os exames foram realizados em analisador hematológico SDH-3 VET ${ }^{\circledR}$. As variáveis hematológicas se mostraram estáveis ao longo do tempo com exceção das hemácias que sofreram variação no T12h em temperatura ambiente e as plaquetas que no T6h em temperatura ambiente e sob refrigeração mantiveramse abaixo do intervalo de referência. Conclui-se que as amostras de cães analisados pela tecnologia de impedância, mais especificamente pelo equipamento labtest SDH-3 VET ${ }^{\circledR}$, mostraram-se estáveis por 48 horas para contagem de hemácias, concentração de hemoglobina, hematócrito e leucócitos totais, porém a contagem de plaquetas deverá ser realizada com amostras colhidas em até 6 horas.

Palavras chave: estabilidade, hematologia, plaquetas

\section{Effect of time and storage temperature on hematological parameters of healthy dogs}

\begin{abstract}
The aim of this study was to evaluate the stability of hematological parameters at different storage times and temperatures. Whole blood from 7 healthy dogs (Canis lupus familiaris) was used. The samples were divided into 2 aliquots of $2 \mathrm{~mL}$ stored in EDTA$\mathrm{K} 3$ tubes, kept at room temperature at $22^{\circ} \mathrm{C}$ and refrigerated at $4^{\circ} \mathrm{C}$, in the following time intervals T0h, T6h, T12h, T24h, T48h. The exams were performed in SDH-3 VET ${ }^{\circledR}$ hematology analyzer. The hematological variables were stable over time with the exception of red blood cells that underwent variation at $\mathrm{T} 12 \mathrm{~h}$ at room temperature and platelets that at $\mathrm{T} 6 \mathrm{~h}$ at room temperature and under refrigeration remained below the reference range. It is concluded that the samples of dogs analyzed by the impedance technology, more specifically by the labtest SDH-3 VET $^{\circledR}$ equipment, were stable for 48 hours for red blood cell count, hemoglobin concentration, hematocrit and total leukocytes; however, the platelets should be performed with samples taken within 6 hours.
\end{abstract}

Keywords: stability, haematological, platelets

\section{Efecto del tiempo de almacenamiento y la temperatura en los parámetros hematológicos de perros sanos}

Resumen. El objetivo de este trabajo fue evaluar la estabilidad de los parámetros hematológicos a diferentes tiempos de almacenamiento y temperaturas. Se utilizó sangre 
total de 7 perros sanos (Canis lupus familiaris). Las muestras se dividieron en 2 partes alícuotas de $2 \mathrm{ml}$ almacenadas en tubos EDTA-K3, mantenidas a temperatura ambiente a $22^{\circ} \mathrm{C}$ y refrigeradas a $4^{\circ} \mathrm{C}$, en los siguientes intervalos de tiempo T0h, T6h, T12h, T24h, T48h. Los exámenes se realizaron en un analizador de hematología labtest SDH-3 VET ${ }^{\circledR}$. Las variables hematológicas fueron estables en el tiempo con la excepción de los glóbulos rojos que experimentaron variación a $\mathrm{T} 12 \mathrm{~h}$ a temperatura ambiente y las plaquetas que a T6h a temperatura ambiente y bajo refrigeración permanecieron por debajo del rango de referencia. Se concluye que las muestras de perros analizadas por la tecnología de impedancia, más específicamente por el equipo SDH-3 VET ${ }^{\circledR}$, fueron estables durante 48 horas para el recuento de glóbulos rojos, la concentración de hemoglobina, el hematocrito y los leucocitos totales, sin embargo, el recuento de plaquetas debe realizarse con muestras recogidas dentro de las 6 horas.

Palabras clave: estabilidad, hematología, plaquetas

\section{Introdução}

Os analisadores hematológicos modernos permitem não só uma avaliação quantitativa e qualitativa precisa das células sanguíneas, mas também fornecem uma vasta gama de parâmetros hematológicos que podem ser úteis para a avaliação diagnóstica e prognóstica de muitas doenças das células sanguíneas (Lippi et al., 2006).

O hemograma é o exame laboratorial mais utilizado na rotina da medicina veterinária (Furlanello et al., 2006; Harvey, 2012); porém, para que o resultado seja confiável é necessário que o exame seja executado respeitando as fases pré-analítica, analítica e pós analítica. A fase pré-analítica envolve todas as atividades que precedem o ensaio, dentro delas está o tempo e a forma de estocagem (Riley, 1992).

Segundo Imeri et al. (2008), o impacto do tempo e da temperatura de armazenamento das amostras antes da análise, deve ser considerado um dos pilares para aumentar a qualidade dos testes hematológicos e melhorar a interpretação clínica dos dados obtidos com diferentes analisadores, e também das diferentes tecnologias e inovação dos equipamentos (Zini, 2013). O processamento exato do hemograma é fundamental para um correto diagnóstico, tratamento e acompanhamento de pacientes (Müller, 2000; Narayanan, 2000). Portanto, torna-se imprescindível conhecer as alterações que o tempo de armazenamento e a temperatura podem ocasionar nas amostras, evitando interpretações equivocadas.

Desta forma, o objetivo deste trabalho foi avaliar o efeito do tempo e da temperatura de armazenamento nos parâmetros hematológicos de cães hígidos processados pela tecnologia de impedância no analisador hematológico SDH-3 VET ${ }^{\circledR}$.

\section{Material e métodos}

Foram utilizadas amostras sanguíneas de 7 cães clinicamente saudáveis independente da raça, sexo e idade. As amostras foram acondicionadas em tubos com anticoagulante EDTA K3 (àcido etilenodiamino tetracetico de potássio) e divididas em duas alíquotas de $2 \mathrm{~mL}$. Uma amostra foi mantida a temperatura ambiente controlada de $22^{\circ} \mathrm{C}$ e a outra conservada em refrigeração a $4^{\circ} \mathrm{C}$. As amostras foram processadas no analisador de células sanguíneas SDH-3 VET ${ }^{\circledR}$ em cinco tempos TOh (até 2 horas após colheita), T6h, T12h, T24h, T48h. Foram mensurados os seguintes parâmetros: contagem total de hemácias $(\mathrm{He})$, concentração de hemoglobina $(\mathrm{Hb})$, hematócrito $(\mathrm{Ht})$, plaquetas (Plt), contagem de leucócitos totais (LT).

Os dados obtidos foram submetidos a análise de variância e comparação das médias pelo teste de Tukey a 5\% de probabilidade pelo método estatística SISVAR (Ferreira, 2011).

\section{Resultados e discussão}

Os resultados das análises do efeito do tempo e temperatura de armazenamento estão expostos na tabela 1. Não houve diferença estatística significativa para os analitos, Hematócrito, Hemoglobina e Leucócitos totais testados em temperatura ambiente controlada $\left(22^{\circ} \mathrm{C}\right)$ e refrigerada $\left(4^{\circ} \mathrm{C}\right)$, mantendo-se estáveis 
até 48 horas pós colheita. Com relação a contagem de hemácias verificou-se variação em temperatura a $22^{\circ} \mathrm{C}$ no $\mathrm{T} 12$ horas, mostrando maior estabilidade em amostras refrigeradas a $4^{\circ} \mathrm{C}$.

Tabela 1. Médias dos parâmetros hematológicos 'de cães analisados no contador hematológico SDH-3 Vet ${ }^{\circledR,}$ em diferentes intervalos de tempo $\left(0,6,12,24\right.$ e 48 horas pós colheita) em temperatura ambiente de $22^{\circ} \mathrm{C}$ e sob refrigeração a $4^{\circ} \mathrm{C}$.

\begin{tabular}{llllllccccc}
\hline \multirow{2}{*}{ Parâmetros } & \multicolumn{9}{c}{$22^{\circ} \mathrm{C}$} \\
\cline { 2 - 11 } & $0 \mathrm{~h}$ & $6 \mathrm{~h}$ & $12 \mathrm{~h}$ & $24 \mathrm{~h}$ & $48 \mathrm{~h}$ & $0 \mathrm{~h}$ & $6 \mathrm{~h}$ & $12 \mathrm{~h}$ & $24 \mathrm{~h}$ & $48 \mathrm{~h}$ \\
\hline $\mathrm{Ht}(\%)$ & 41,64 & 42,94 & 42,68 & 42,4 & 44,44 & 41,64 & 43,84 & 44,08 & 44,08 & 44,37 \\
$\mathrm{Hb}(\mathrm{g} / \mathrm{dL})$ & 14,45 & 13,92 & 13,97 & 14,68 & 14,7 & 14,45 & 14,14 & 14,42 & 14,57 & 14,78 \\
$\mathrm{He} \times 10^{6} / \mathrm{mm}^{3}$ & 6,14 & 6,47 & $5,35^{\mathrm{a}}$ & 6,38 & 6,34 & 6,14 & 6,35 & $6,41^{\mathrm{b}}$ & 6,47 & 6,3 \\
Leu x $10^{3} / \mathrm{mm}^{3}$ & 10,4 & 10,3 & 8,8 & 10,4 & 10,4 & 10,4 & 10,5 & 10 & 11.2 & 10,9 \\
Plt x $10^{3} / \mathrm{mm}^{3}$ & $380^{\mathrm{b}}$ & $216,8^{\mathrm{a}}$ & $190,1^{\mathrm{a}}$ & $175,5^{\mathrm{a}}$ & $155,4^{\mathrm{a}}$ & $380^{\mathrm{b}}$ & $149,1^{\mathrm{a}}$ & $169,1^{\mathrm{a}}$ & $135,4^{\mathrm{a}}$ & $162,7^{\mathrm{a}}$ \\
\hline
\end{tabular}

a,b Letras diferentes nas linhas diferem estatisticamente pelo teste Tukey 5\%. Ht-hematócrito, Hb-concentração de hemoglobina, He-contagem total de hemácias, Leu-leucócitos totais, Plt-plaquetas.

Somente a contagem das plaquetas apresentaram diferença estatística significativa dentro dos períodos avaliados, com contagens menores a partir das 6 horas, com valores abaixo da referência, caracterizando uma pseudotrombocitopenia, sugestivo de um fenômeno in vitro resultante da aglomeração plaquetária secundária a venopunção e exposição do sangue ao anticoagulante EDTA (Weiss \& Wardrop, 2010). Fato este que mostra a importância da avaliação criteriosa do esfregaço sanguíneo por microscopia óptica, pois estará evidente a presença de agregação plaquetária. Lee \& Kang (2016) também verificaram alterações significativas na contagem de plaquetas em amostras sanguíneas de cães, no tempo de 24 horas, porém não avaliaram em intervalos menores que 24 horas, deixando em dúvida se esta alteração poderia ter ocorrido antes a esse tempo.

Buoro et al. (2016) estudaram amostras de sangue humano e concluíram que o ideal é realizar o hemograma em até 2 horas após a colheita, entretanto consideraram que o máximo de tempo entre a colheita e a análise seria de 8 horas. Já Cora et al. (2012) verificaram em amostras de sangue de ratos, a estabilidade na concentração de hemácias e hemoglobina de até 72 horas, com analisador hematológico que utiliza a impedância na contagem total de células, bem como no seu tamanho. Porém verificaram aumento do hematócrito coincidindo com aumento no Volume Corpuscular Médio (VCM) em até 6 horas pós colheita.

Médaille et al. (2006) não encontraram variações para hemácias e hemoglobina em amostras de cães, entretanto verificaram alterações para a contagem de leucócitos totais e plaquetas, com diminuição significativa nas 24 horas, e relataram que estas variações devem ser conhecidas para evitar erros na interpretação.

Estes resultados mostraram a importância do conhecimento e validação tecnológica dos equipamentos utilizados em laboratórios clínicos a fim de que se possa trabalhar com mais segurança nos resultados obtidos.

\section{Conclusão}

A estabilidade das hemácias e das plaquetas variam com o tempo e temperatura de estocagem, portanto amostras de sangue de cães analisados pela tecnologia de impedância, mais especificamente pelo equipamento SDH-3 VET $^{\circledR}$, devem ser processadas de preferência com sangue fresco ou em menos de 6 horas, e mantidas sob refrigeração a $4^{\circ} \mathrm{C}$.

\section{Referências bibliográficas}

Buoro, S., Mecca, T., Azzarà, G., Seghezzi, M., Dominoni, P., Crippa, A., Ottomano, C., \& Lippi, G. (2016). Cell population data and reflex testing rules of cell analysis in pleural and ascitic fluids using body fluid mode on Sysmex XN-9000. Clinica Chimica Acta, 452, 92-98. DOI: https://doi.org/10.1016/j.cca.2015.11.005

Cora, M. C., King, D., Betz, L. J., Wilson, R., \& Travlos, G. S. (2012). Artifactual changes in spraguedawley rat hematologic parameters after storage of samples at $3 \mathrm{C}$ and 21 C. Journal of the American 
Association for Laboratory Animal Science, 51(5), 616-621.

Ferreira, D. F. (2011). SISVAR: A Computer Statistical Analysis System. Ciência e Agrotecnologia, 35(6), 1039-1042. DOI: http://dx.doi.org/10.1590/S1413-70542011000600001

Furlanello, T., Tasca, S., Caldin, M., Carli, E., Patron, C., Tranquillo, M., Lubas, G., \& Solano-Gallego, L. (2006). Artifactual changes in canine blood following storage, detected using the ADVIA 120 hematology analyzer. Veterinary Clinical Pathology, 35(1), 42-46. DOI: https://doi.org/10.1111/j.1939-165X.2006.tb00087.x

Harvey, J. W. (2012). Veterinary hematology: A diagnostic guide and color atlas. In Louis, Missouri: Elsevier Saunders. Elselvier Saunders.

Imeri, F., Herklotz, R., Risch, L., Arbetsleitner, C., Zerlauth, M., Risch, G. M., \& Huber, A. R. (2008). Stability of hematological analytes depends on the hematology analyser used: a stability study with Bayer Advia 120, Beckman Coulter LH 750 and Sysmex XE 2100. Clinica Chimica Acta, 397(1-2), 68-71. DOI: https://doi.org/10.1016/j.cca.2008.07.018

Lee, J.-M., \& Kang, J. S. (2016). Changes of hematological references depends on storage period and temperature conditions in rats and dogs. Laboratory Animal Research, 32(4), 241-248. DOI: https://doi.org/10.5625/lar.2016.32.4.241

Lippi, G., Guidi, G. C., Mattiuzzi, C., \& Plebani, M. (2006). Preanalytical variability: the dark side of the moon in laboratory testing. Clinical Chemistry and Laboratory Medicine, 44(4), 358-365. DOI: https://doi.org/10.1515/CCLM.2006.073

Médaille, C., Briend-Marchal, A., \& Braun, J.-P. (2006). Stability of selected hematology variables in canine blood kept at room temperature in EDTA for 24 and 48 hours. Veterinary Clinical Pathology, 35(1), 18-23. DOI: https://doi.org/10.1111/j.1939-165X.2006.tb00083.x

Müller, M. M. (2000). Implementation of reference systems in laboratory medicine. Clinical Chemistry, 46(12), 1907-1909. DOI: https://doi.org/10.1093/clinchem/46.12.1907

Narayanan, S. (2000). The preanalytic phase: an important component of laboratory medicine. American Journal of Clinical Pathology, 113(3), 429-452. DOI: https://doi.org/10.1309/c0nm-q7r0-112e-b3uy

Riley, J. H. (1992). Clinical pathology: Preanalytical variation in preclinical safety assessment studieseffect on predictive value of analyte tests. Toxicologic Pathology, 20(3-2), 490-500. DOI: https://doi.org/10.1177/0192623392020003206

Weiss, D. J., \& Wardrop, J. K. (2010). Schalm's Veterinary Hematology.

Zini, G. (2013). Stability of complete blood count parameters with storage: toward defined specifications for different diagnostic applications. International Journal of Laboratory Hematology, 36(2), 111-113. DOI: https://doi.org/10.1111/ijlh.12181

Recebido: 22 de abril, 2020. Aprovado: 28 de maio, 2020

Disponível online: 8 de outubro, 2020.
Licenciamento: Este artigo é publicado na modalidade Acesso Aberto sob a licença Creative Commons Atribuição 4.0 (CC-BY 4.0), a qual permite uso irrestrito, distribuição, reprodução em qualquer meio, desde que o autor e a fonte sejam devidamente creditados. 